\title{
Integrated transmitter devices on InP exploiting electro-absorption modulation
}

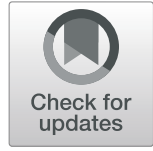

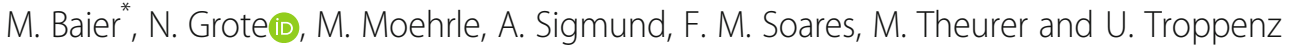

\author{
* Correspondence: moritz.baier@hhi. \\ fraunhofer.de \\ Fraunhofer Institute for \\ Telecommunications, Heinrich Hertz \\ Institute, Einsteinufer 37, 10587 \\ Berlin, Germany
}

\begin{abstract}
InP technology is the principal enabler for implementing fully monolithic photonic integrated circuits (PIC), uniquely including transmitter elements. In this article we present an overview of recent achievements on ultra-high speed electro-absorption modulated lasers (EML) which represent a simple transmitter PIC comprising a singlemode laser diode and an electro-absorption modulator. Using a so-called identicallayer approach single-wavelength modulation rates up to $100 \mathrm{~Gb} / \mathrm{s}$ have been accomplished. By additionally integrating an optical amplifier section modulated optical output power of $>10 \mathrm{dBm}$ has been achieved. Multi-level amplitude modulation was successfully demonstrated. Extended EML chips designed for wavelength-division and space-division multiplexing, respectively, will be presented. For dual-polarization transmission a novel EML related transmitter as well as a corresponding receiver PIC have been introduced. The latter devices were made on a generic PIC platform that is available for open-access foundry service.

Keywords: Electro-absorption modulated laser, Booster amplifier, $100 \mathrm{~Gb} / \mathrm{s}$ modulation, Photonic integrated circuit, Building block, Polarization multiplexing, Polarization sensitive receiver
\end{abstract}

\section{Introduction}

Photonic integrated circuits (PIC) have been evolving over the past 10 years to progress from the research to the commercial stage. A recent market study predicts a market volume of 3.3 bn USD by the year 2027 [1]. Photonic integration has already been a subject of research since the 1980's using optically passive waveguide materials, such as $\mathrm{SiO}_{2}$, but particularly InP along with the related compound semiconductor materials InGaAsP and InGaAlAs. Their natural wavelength range of operation of about 1300$1650 \mathrm{~nm}$ perfectly matches the optimal spectral range of optical fibers used for fiber optic communications. Beyond passive-optical functionalities this class of material is as well capable of providing active optical functions, namely laser light generation and detection, making it a perfect choice for full monolithic integration. Since the 1990's also silicon has been investigated as a photonic material, using silicon-on-insulator (SOI) as base material nowadays. Because $\mathrm{Si}$ is optically transparent at wavelengths above $1150 \mathrm{~nm} \mathrm{Ge}$ is incorporated by hetero-epitaxial techniques to implement photodetector devices. The very principal difference between InP and Si photonics is, however, the fact that lasers and associated devices (semiconductor optical amplifiers, gain chips) cannot be efficiently made on $\mathrm{Si}$ - and there are no short or medium-term 
solutions in sight. Instead, InP based lasers need to be integrated using sophisticated nonmonolithic techniques ranging from simple fiber connection, hybrid micro-assembly and pick-and-place mounting, and transfer printing to heterogeneous integration by wafer bonding and epitaxial growth. Hence, Si photonics represents a technology mix of monolithic and hybrid integration (see e.g. [2, 3]).

In contrast, InP technology enables laser integration in a fully monolithic manner, rendering photonic integration on InP particularly favorable when transmitter functionalities play a dominant role. In this article we summarize recent results on integrated transmitter devices exploiting electro-absorption modulation that have been achieved at the Fraunhofer Heinrich Hertz Institute (HHI).

\section{Electro-absorption modulated laser structures}

A (seemingly) simple transmitter PIC is an electro-absorption modulated laser (EML) which basically represents the monolithic integration of two different elements: a highspeed electro-absorption modulator (EAM) and a single-wavelength cw-driven laser source. By exploiting the Quantum-Confined Stark effect (QCSE) to change the internal absorption EAMs can principally be modulated at ultra-high speed, mainly limited by carrier transport issues. In any case maximum achievable modulation rates are much beyond those of directly modulated laser diodes the modulation rate of which is practically restricted to the hierarchical bit rate level of $25 / 28 \mathrm{~Gb} / \mathrm{s}$. To accomplish EML devices different integration schemes have been applied recently, as illustrated in Fig. 1: (a) butt-joint coupling; (b) identical layer structure; and (c) waveguide interconnection.

The principal advantage of approach (a) is the fact that the structure of both constituent devices can be separately optimized, e.g. the active multi-quantum layers (MQW) may be made of InGaAsP for the laser and of InGaAlAs for the EAM section. The latter favours rapid carrier sweep-out from the MQW region when switching from the absorption to the transparency state thus guaranteeimg superior ultra-high speed modulation. InGaAsP-containing laser structures, on the other hand, are known for their uncritical reliability behaviour. The fabrication of butt-joint structures is, however, more demanding and the interface may cause significant internal back-reflection of the laser light. Nowadays, several commercial manufacturers of EMLs are using the buttcoupling approach.

The main feature of the identical-layer (IL) approach (b) is its fabrication simplicity. Apart from etching a groove to electrically isolate the laser and EAM sections device

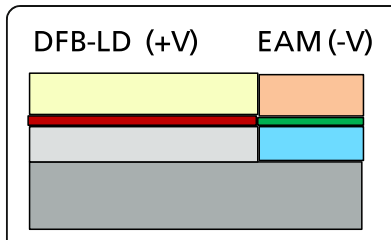

(a)

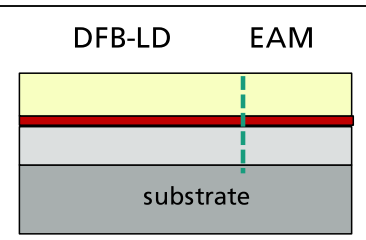

(b)

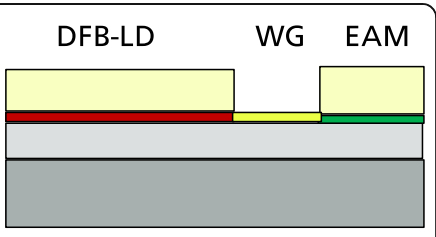

(c)

Fig. 1 Principal integration schemes for EML components (DFB-LD = distributed feedback laser diode; EAM = electro-absorption modulator; $W G=$ optical waveguide; $V=$ voltage) 
processing is pretty much the same as with ordinary DFB laser diodes. Therefore, the IL scheme opens the way to achieving very low-cost devices. The structure provides smooth optical transition between laser and EAM virtually eliminating internal reflections. On the other side, as the band-edge is the same across the entire EML structure the DFB laser wavelength has to be set (via the period of the DFB grating) on the longwavelength tail of the gain spectrum to guarantee low residual absorption inside the EAM during the on-state. Consequently, higher drive currents are required for reaching a given level of optical laser power.

(c) Optically interconnecting the laser and the EA modulator by a waveguide represents a logical step to utilize electro-absorption modulation in extended photonic integrated circuits. Such an approach requires, however, a more comprehensive and even more demanding integration technology, as developed at HHI (see sect. 5). A unique fabrication technique has been applied recently exploiting special epitaxial growth effects on masked substrates, referred to as selective-area-growth (SAG). With this method local bandgap engineering can be accomplished to enable individually optimizing the active bandgap wavelengths of the laser, EAM, and waveguide in a single epitaxial step. A related transmitter PIC for generating complex amplitude and phase modulation formats has been presented e.g. in [4].

\section{Identical-layer EML devices}

At Fraunhofer HHI there is a long-standing expertise on EMLs using the IL device concept [5]. Typical performance parameters of HHI's EML devices, consisting of a $300 \mu \mathrm{m}$ long DFB laser and an $80 \ldots 120 \mu \mathrm{m}$ long EAM section and containing an InGaAlAs-MQW active region on n-doped substrate (Fig. 2), are characterized by a static extinction ratio of $>20 \mathrm{~dB}$ at an EAM reverse voltage change of $-2 \mathrm{~V}$. The typical bias voltage around which the EAM is modulated is about $-1 \mathrm{~V}$. Below this point the chirp factor tends to become zero, and even negative when further shifting the bias towards larger negative values. The optical output power measured at $0 \mathrm{~V}$ (short circuit) reaches some $5 \mathrm{dBm}$. This limit is mainly given by the maximum power level that the EAM can handle particularly at high modulation speed. To some extent it also accounts for the fact that the DFB laser is not operated at optimum gain but is detuned

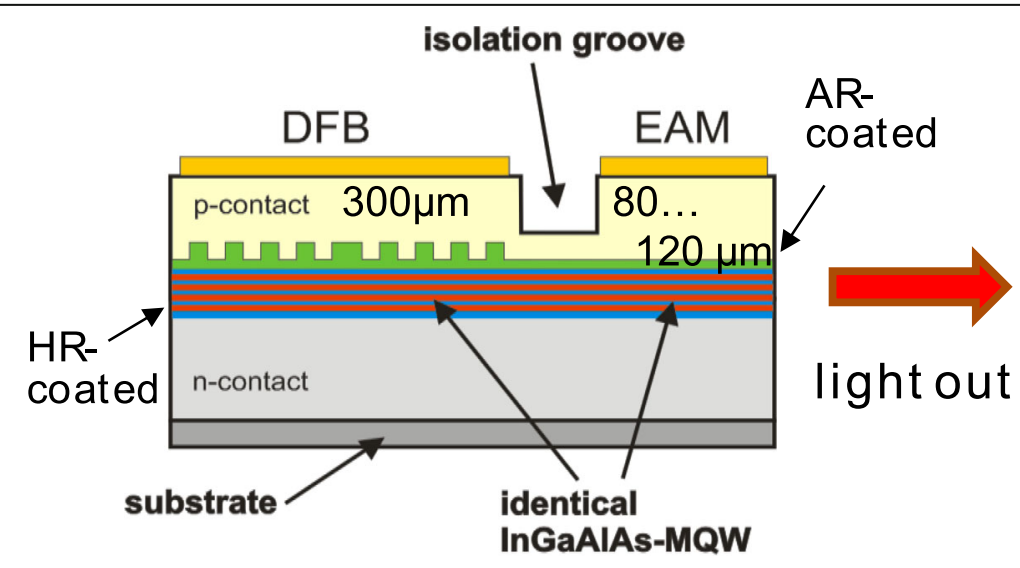

Fig. 2 Schematic cross-section of EML device using an identical layer stack 
to longer wavelengths to guarantee transparency of the electro-absorption modulator in the on-state.

To overcome this design-inherent issue an optical booster amplifier has been successfully added by simply forming another isolation groove (Fig. 3). This extended EML structure readily provides output powers of $>10 \mathrm{dBm}$. Even at $56 \mathrm{~Gb} / \mathrm{s}$ the integrated amplifier was found not to affect the modulation behavior [5]. Thanks to the booster upgrade next-generation PON requirements (average modulated output power of min $9 \mathrm{dBm} @ 10 \mathrm{~Gb} / \mathrm{s} ; 1577 \mathrm{~nm})$ can readily be met.

The modulation rate of $56 \mathrm{~Gb} / \mathrm{s}$ is routinely achievable now [6]. However even a bit rate of $100 \mathrm{~Gb} / \mathrm{s}$ has been demonstrated recently (Fig. 4) after introducing further design refinements. Hence, a single-wavelength $100 \mathrm{G}$ transmitter (on-off keying) may be implemented using a single tiny EML chip (provided suitable electrical drivers are available).

In addition or instead, however, virtually all the common multiplex methods are applicable to efficiently reach even higher bit rates and/or to exploit lower baud rates. 4- and 8-level Pulsed Amplitude Modulation (PAM) could be successfully demonstrated on HHI's EML devices using a proprietary SiGe based driver IC. Clear 32 GBd PAM-4 eye diagrams have been achieved at a driver power of $84 \mathrm{~mW}$ leading to a figure-of-merit of $1.3 \mathrm{pJ} / \mathrm{bit}$ for the driver (Fig. 5).

For space-division-multiplexed transmission a serial dual-EML chip has been designed that comprises two EAMs in back-to-back configuration sharing the same DFB source laser between them [7]. Simultaneous modulation without mutual interference was demonstrated at $56 \mathrm{GBd}$ delivering a total bit rate of $112 \mathrm{~Gb} / \mathrm{s}$ (Fig. 6).

For parallel single mode (PSM) transmission schemes a parallel 4-EML array was fabricated [8] which is capable of generating an aggregated data rate of $224 \mathrm{~Gb} / \mathrm{s}(4 \times 56$ GBd). As a special feature, in this array the RF connections to the EAMs have been routed to the rear edge of the chip thus providing uniform RF driving conditions and concurrently facilitating packaging (Fig. 7 (left). Evidently, the latter device can also be deployed for wavelength-division-multiplexing (WDM) systems when purposely shifting the DFB wavelengths of the individual EMLs of the array, in the present case by means of e-beam lithography (Fig. 7 (right). Generally, however, the applicability of EMLs to WDM transmission schemes is restricted because the exploited electroabsorption mechanism relies on a non-linear semiconductor band-edge effect. Depending on performance uniformity requirements the same EAM structure may therefore

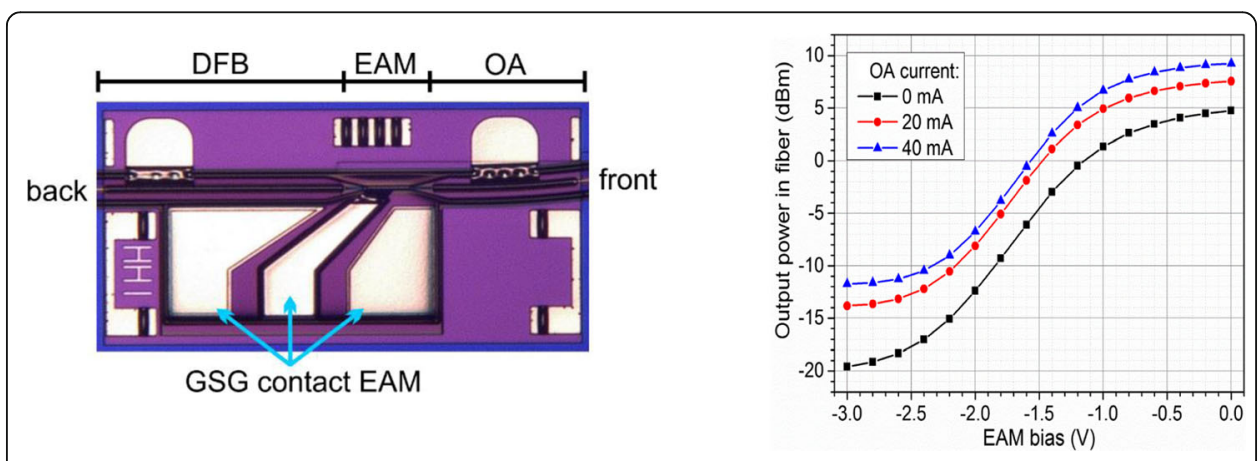

Fig. 3 EML structure extended by an optical booster amplifier (OA) (left); optical output power characteristics as function of EAM bias and booster drive current (right) [5] 


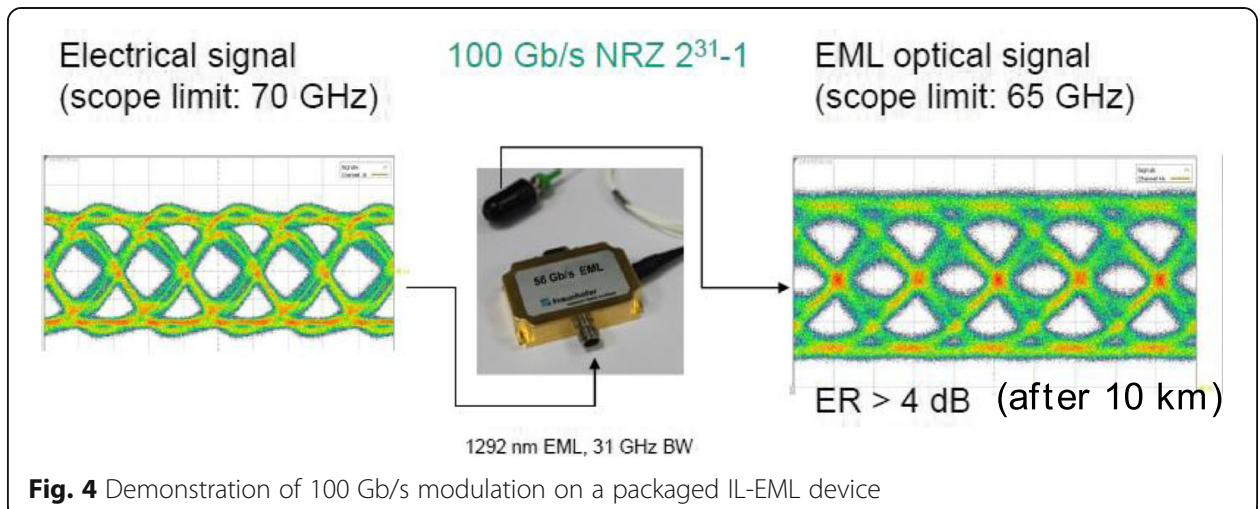

only be usable over a spectral WDM range of 5-10 nm, i.e. essentially for low-channel DWDM systems.

In turn the said wavelength range translates -for a given single wavelength-into an operating temperature range of $50-100 \mathrm{~K}$ in which the EML can be used without temperature control. This mode of operation could be successfully demonstrated recently on HHI's EML devices. Nonetheless, despite these promising results EMLs are commonly operated under TEC controlled conditions yet, typically at $40 \ldots 50^{\circ} \mathrm{C}$ heat-sink temperature.
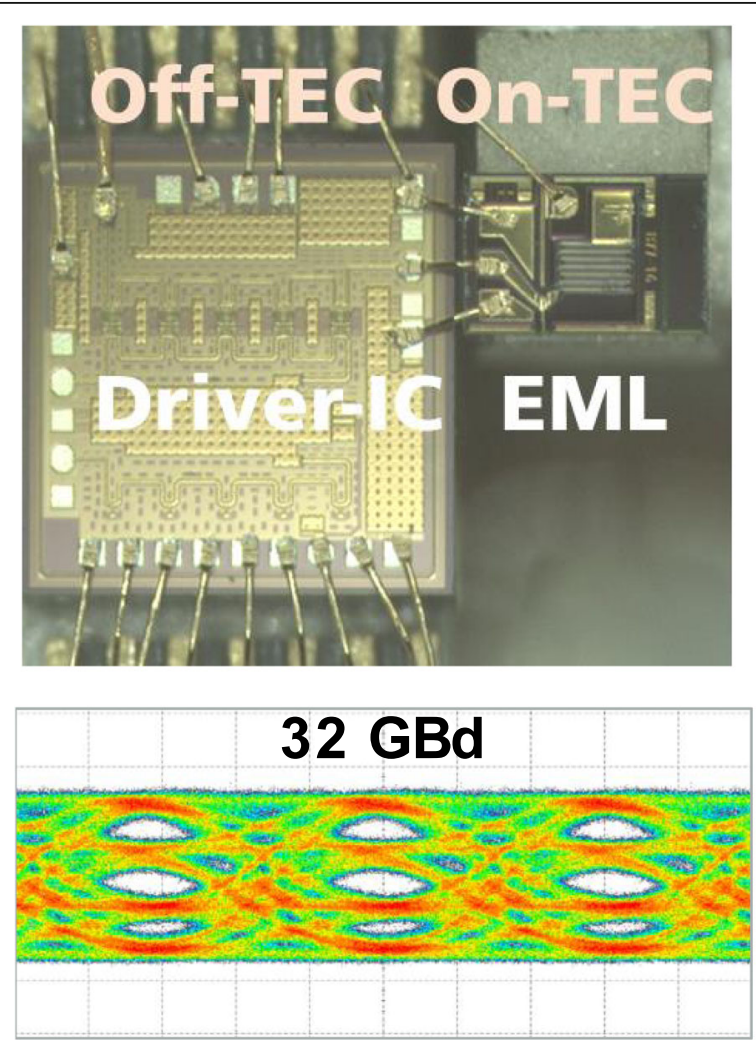

Fig. 5 PAM-4 eye diagram at $32 \mathrm{GBd}$. The driver IC (top) was designed at $\mathrm{HHI}$ and fabricated on the BiCMOS foundry line of the Leibniz-Institut für Innovative Mikroelektronik (IHP), Germany 


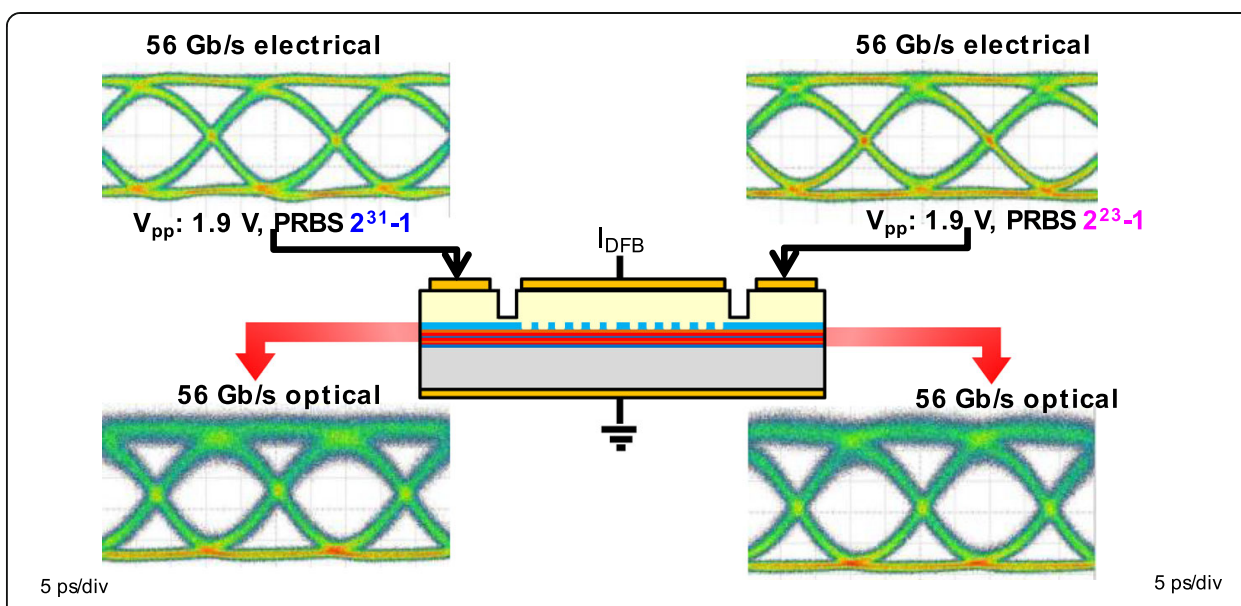

Fig. 6 Configuration of a Dual-EML and input (electrical) and output eye diagrams (optical) at $56 \mathrm{~Gb} / \mathrm{s}$ [7]

\section{EML based polarization multiplexing}

Beyond exploiting the physical parameters wavelength and space the polarization of light is increasingly exploited for signal multiplexing, doubling the bit rate when using the TE and TM state of polarization. This scheme is being widely used with coherent transmission systems using e.g. a Mach-Zehnder modulator based IQ modulator as transmitter. For direct-detection transmission schemes an EML based dual-polarization transmitter laser (DPEML) was recently introduced by HHI $[9,10]$ that essentially comprises two serial EAMs in combination with combiner/splitter-free polarization rotator elements [11] and a DFB laser source. All of them are optically connected by a waveguide making this arrangement a true photonic integrated circuit. Figure 8 shows a functional block diagram together with a fabricated chip, measuring only $3.2 \mathrm{~mm} \times$ $0.2 \mathrm{~mm}$. The EAMs are used to modulate two independent states of polarization (X and $\mathrm{Y}$ ). These are created by a $45^{\circ}$ - polarization rotator that rotates the strongly TEpolarized light of the DFB laser by 45 deg resulting in TE-and TM signal components of equal intensity. As QW-based EAMs are only responding to TE polarized light, the first EAM only modulates the TE signal but lets the TM fraction pass unaffected. The $90^{\circ}$ rotator then converts these two states into TM and TE, respectively, such that the previously TM polarized component can be modulated by the second EAM. To sufficiently suppress cross-modulation the polarization rotator and the EAM devices need

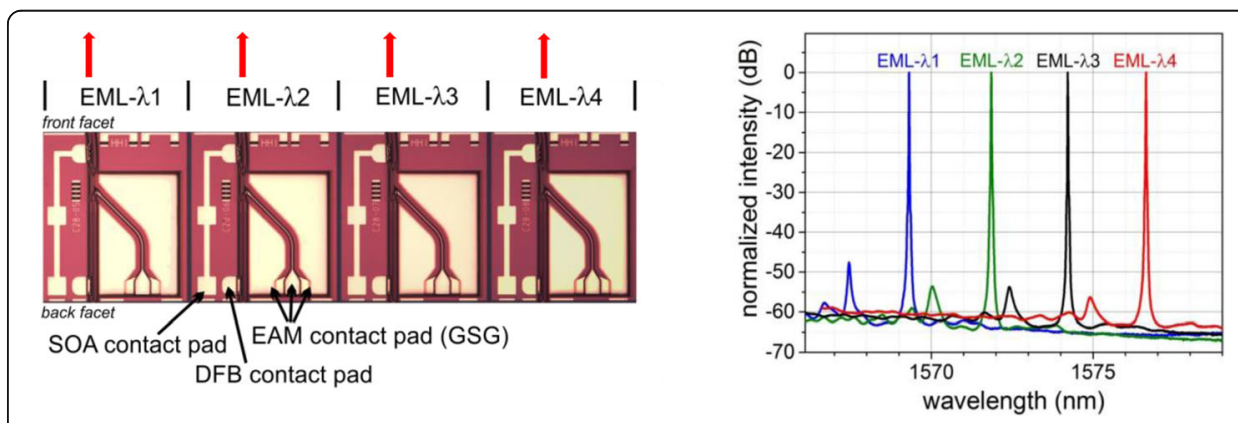

Fig. 7 Monolithic 4-array EML chip with the RF connections of the EAMs equally routed to the rear facet (left), chip dimension: $2545 \mu \mathrm{m} \times 725 \mu \mathrm{m}$; emission spectra of the individual EMLs spaced by $2.5 \mathrm{~nm}$ 


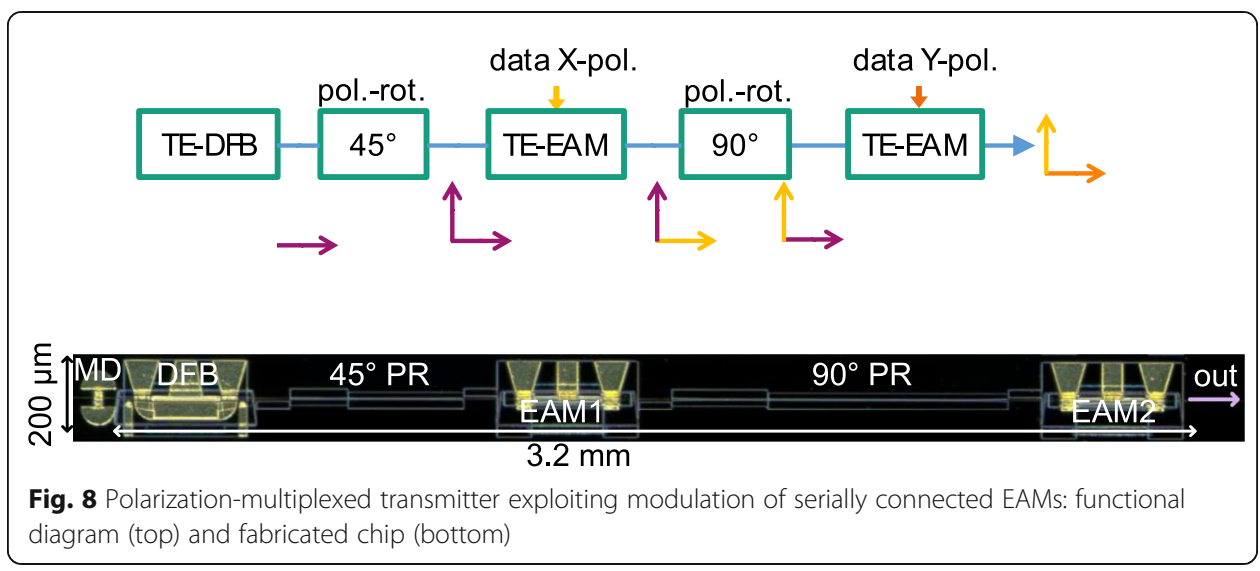

to provide a high TE/TM polarization extinction ratio (PER). Typically the $90^{\circ}$ rotator exhibits a PER value of $>20 \mathrm{~dB}$ across the C-band, and the current EAMs about $20 \mathrm{~dB}$. Evaluation of first chips performed at $20 \mathrm{~Gb} / \mathrm{s}$ has proven the validity of this approach. On a precursor chip without integrated laser, a total bit rate of $112 \mathrm{~Gb} / \mathrm{s}$ was obtained using a baud rate of $28 \mathrm{GBd}$ and PAM-4 modulation (Fig. 9) [9].

By further inserting an optical phase shifter to modulate the optical phase between the TE- and TM-signal, a very compact transmitter for true 3-dimensional Stokesvector modulation (SVM) may be realized delivering a bit rate corresponding to the threefold baud rate, and six-fold if additionally applying PAM-4 signaling to each of the three channels. SVM represents a promising new direct modulate-and-detect technique [12] to be positioned between direct and coherent transmission which may have high potential for $100 \mathrm{G}$, and above, access and metro networks.

A solution for the corresponding receiver [13] is depicted in Fig. 10. It essentially represents a $5 \times 5$-Mach-Zehnder interferometer with $5 \times 5$ multimode interference $(\mathrm{MMI})$ structures used as couplers. On each of the five birefringent waveguide arms between them a polarization rotator (PR) is integrated to perform the function of a circular quarter-wave plate. While the arms are equal in length the rotator devices are positioned differently. This lay-out, in conjunction with the waveguide's birefringence, ensures the interference condition at the output coupler to be strongly polarization dependent. The respective signals are converted into currents by integrated

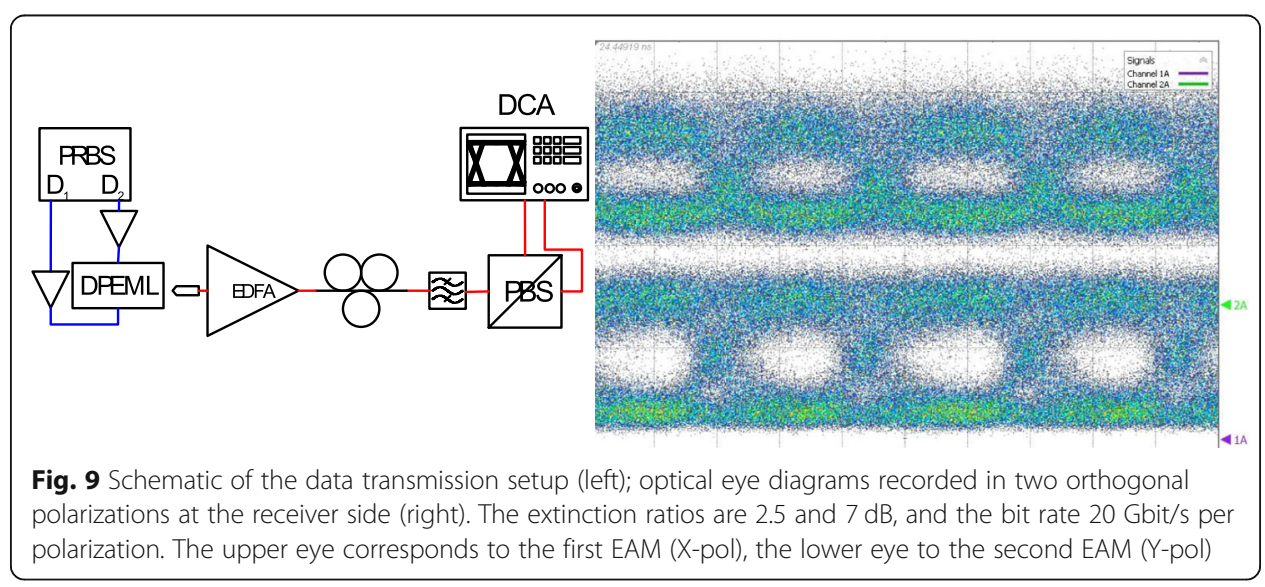



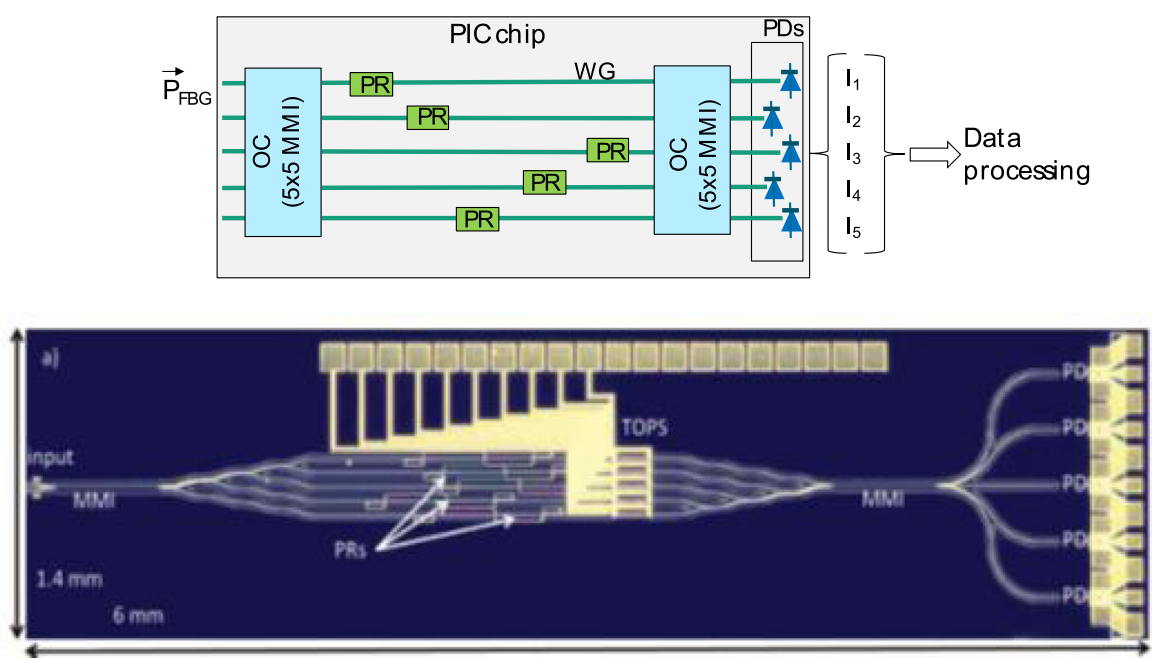

Fig. 10 Architecture of polarization-sensitive receiver (top) and fabricated chip (bottom); (OC = optical coupler; $\mathrm{PR}=$ polarization rotator; $\mathrm{WG}=$ waveguide; $\mathrm{PD}=$ photodiode; $\mathrm{I}_{\mathrm{x}}=$ photocurrent)

photodiodes from which the original state-of-polarization (SOP) of the received light can be retrieved.

Figure 11 shows an experimental result of early devices at $1550 \mathrm{~nm}$ wavelength. The blue dots represent states-of-polarization of the light launched into the chip via an optical fiber, and the red dots the measured SOPs. The average error between these points was found to be less than $1 \mathrm{deg}$, with a maximum value of $4 \mathrm{deg}$.

\section{PIC foundry platform}

While the EML devices addressed in sect. 3 rely on dedicated layer structures - adapted to O-, C- and L-band operation, respectively -, the previous PIC devices were realized on a generic monolithic integration platform that has been developed at HHI over the past years [14]. Building on semi-insulating InP substrate (currently 3-in.) and on InGaAsP/InP material (no InGaAlAs included yet) this platform is capable of integrating transmitter, receiver and passive-optical functional elements (“TxRx-platform").
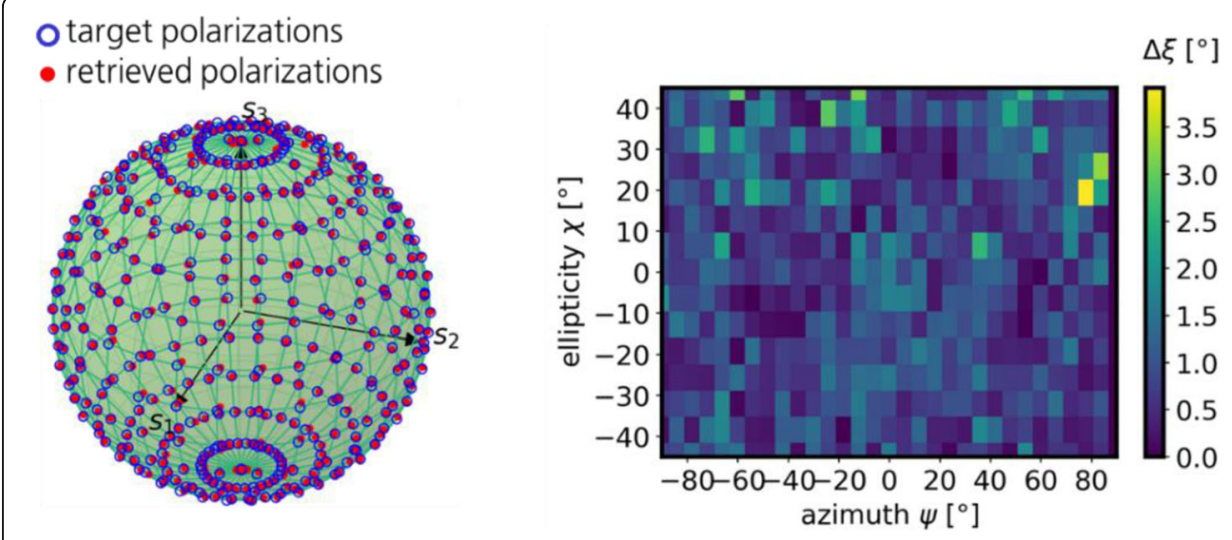

Fig. 11 Experimental results of a fabricated device at the wavelength $1550 \mathrm{~nm}$. The blue dots represent states of polarization (SOPs) that are launched into the chip by a tapered fiber. The red dots are the measured SOPs (left); angular distance $\Delta \xi$ between launched SOPs and measured dots (right) 
Table 1 Building blocks available on Fraunhofer HHI's PIC foundry platform

\begin{tabular}{lll}
\hline Tx-type BB & Passive BB & Rx-type BB \\
\hline gain element/SOA & optical waveguide (3 different index contrasts) & pin-PD (40G \& <10G) \\
DFB laser diode (20G) & tapered waveguide transition & PD impedance matching RC \\
DBR laser & waveguide circular arc & \\
DBR laser & waveguide crossing & \\
phase shifter (current inject.) & I/O spot size converter & \\
tunable Bragg grating & directional coupler \\
& MMl (1 × 2; 2×2) \\
& arrayed waveguide grating \\
& polarisation converter \\
& polarisation beam splitter \\
& polarisation converter \\
& phase shifter (thermo- optic) \\
& metal interconnects \& waveguide crossover \\
& RF lines \\
& p - isolation section \\
&
\end{tabular}

Designed for C-band wavelengths a comprehensive set of (partly parameterized) building blocks is available from which designers can choose to devise their own PICs (Table 1). The required "Process Design Kit (PDK)" is provided by currently five companies offering design software packages for PIC lay-out, physical and circuit-level simulation to automated generation of mask files.

HHI has been offering a foundry service since 2014 to fabricate such PICs on scheduled multi-project wafer (MPW) runs (currently 4 wafer starts per year). Thanks to the generic nature of the platform these runs may be shared by up to 14 users thus rendering InP based PIC prototype chips more than affordable. In Fig. 12 an example is shown.

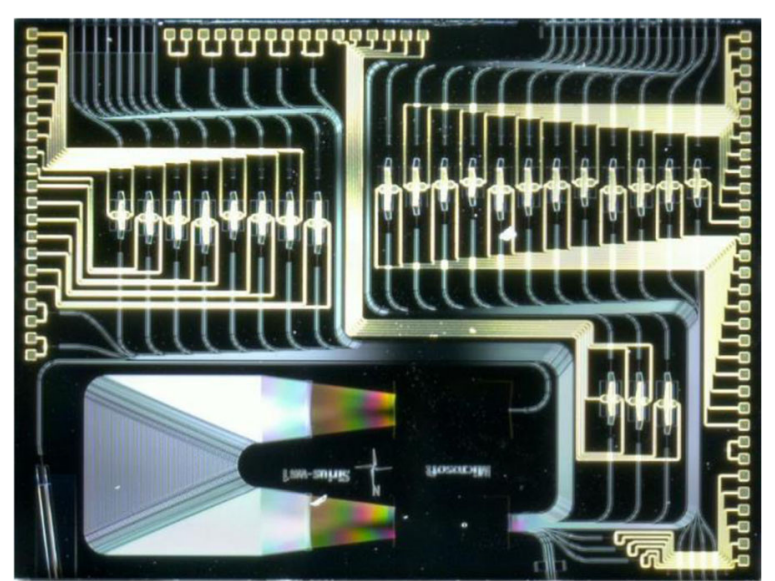

Fig. 12 Photograph of an exemplary (published) InP PIC chip made on a foundry MPW run at Fraunhofer $\mathrm{HHI}$. The PIC which, amongst others, incorporates a number optical amplifiers was designed by Microsoft for sub-ns wavelength switching applications [15] 


\title{
Conclusion
}

InP provides a PIC platform that supports true monolithic integration of transmitter related devices, namely lasers and gain elements/optical amplifiers. Therefore InP represents the integration technology of choice when transmitter functions are involved. A simple transmitter PIC is realized by integrating a single-mode source laser and an electro-absorption modulator. Exploiting a very cost-effective embodiment based on an identical-layer structure bit rates of up to $100 \mathrm{~Gb} / \mathrm{s}$ could be accomplished using simple amplitude modulation at a single wavelength. Given respective driver ICs are available, such devices are highly suited for simplified, lower cost $100 \ldots 400 \mathrm{G}$, and beyond, transceivers for data centers. Using a generic PIC platform dual-polarization EML components have been introduced integrating a laser, polarization rotators and electro-absorption modulators, all of them serially connected by an optical waveguide. These components in conjunction with corresponding receiver PICs may pave the way for applying StokesVector modulation, a potentially low-cost approach positioned between direct modulation and coherent transmission techniques, to high-speed optical data systems.

\begin{abstract}
Abbreviations
(D)WDM: (Dense) wavelength division multiplex; (DP)EML: (Dual-polarization) electro-absorption modulated laser; BiCMOS: Bipolar complementary metal-oxide-semiconductor; DFB-LD: Distributed feedback - laser diode; EAM: Electroabsorption modulator; G: Gigabit; HHI: Heinrich Hertz Institute; IC: Integrated circuit; IL: Identical-layer; InGaAlAs: Indium-gallium-aluminium-arsenide; InGaAsP: Indium-gallium-arsenide-phosphide; InP: Indiumphosphide; MMI: Multimode interference; MPW: Multi-project wafer; MQW: Multi-quantum well; OA: Optical amplifier; OC: Optical coupler; PAM: Pulsed amplitude modulation; PD: Photodiode; PDK: Process design kit; PER: Polarization extinction ratio; PIC: Photonic integrated circuit; PON: Passive optical network; PR: Polarization rotator; PSM: Parallel single mode; QCSE: Quantum confined Stark effect; SAG: Selective-area-growth; SOI: Silicon-on-isolator; SOP: State-of-polarization; SVM: Stokes-vector modulation; TE: Transverse electric; TEC: Thermos-electric cooler; TM: Transverse magnetic; WG: Waveguide
\end{abstract}

\section{Acknowledgements}

Many more colleagues contributed, in one way or another, to the work leading to the reviewed results. Mainly they have been acknowledged in the referenced publications.

\section{Authors'contributions}

NG: principal writer of the article; former supervisor of research activities having led to the described results. FS: principal developer of generic PIC platform at Fraunhofer HHI, respective text contributions. MB: conceptual and design work and text contributions for dual-polarization Transmitter PIC and related Receiver PIC described in the article. MM: principal developer and supervisor of EML device activities described in the article. MT, UT: design, technology development and EML device evaluation; generation of related figures. AS: EML process development and device fabrication. All authors read and approved the final manuscript.

\section{Funding}

For the development of the generic PIC platform outlined in the article funding has been received from the European Commission through the projects EuroPIC (www.europic.jeppix.eu); PARADIGM (www.paradigm.jeppix.eu), and InPulse (www.inpulse.jeppix.eu). The 4-array EML chips (refer to Fig. 7) was developed in the framework of the PIANO+-ALOHA project (grant \# 01BP1125). The other topics have been conducted on HHI internal funding.

\section{Availability of data and materials}

The dataset(s) supporting the conclusions of this article is (are) included within the article (and its additional file(s))."

\section{Competing interests}

The authors declare that they have no competing interests.

Received: 10 October 2019 Accepted: 29 December 2019

\section{0.010}

\section{References}

1. Photonic Integrated Circuits Market to Reach $\$ 3.3$ Billion by 2027. 2019. https://www.novuslight.com/photonicintegrated-circuits-market-to-reach-3-3-billion-by 2027_N9494.html

2. Mack M, Peterson M, Gloeckner S, Narasimha A, Koumans R, Dobbelaere PD. Method and system for a light source assembly supporting direct coupling to an integrated circuit, US8168939B2; 2012.

3. Wang Z, Abbasi A, Dave U, Kurnari S, Kunert B, Merckling C, Pantouvaki M, Shi Y, Tian B, Van Gasse K, Verbist J, Wang R, Xie W, Zhang J, Zhu Y, Bauwelinck J, Yin X, Hens Z, Van Campenhout J, Kuyken B, Baets R, Morthier G, Van Thourhout D, Roelkens G. Novel light source integration approaches for silicon photonics. Laser Photonics Rev. 2017;11:1-21. 
4. Kazmierski C, Chimot N, Jorge F, Konczykowska A, Blache F, Decobert J, Alexandre F, Garreau A, da Silva R. 80 Gb/s multi-level BPSK experiment with an InP monolithic source based on prefixed optical phase switching, 26th IPRM (Montpellier, France), paper Th-B2-1; 2014.

5. Moehrle M, Klein H, Bornholdt C, Przyrembel G, Sigmund A, Molzow W-D, Troppenz U, Bach H-G. InGaAlAs RW-based electro-absorption-modulated DFB-lasers for high speed applications, SPIE photonics Europe 2014, Brussels, invited paper 9134-44; 2014

6. Theurer M, Przyrembel G, Sigmund A, Molzow WD, Troppenz U, Möhrle M. 56 Gb/s L-band InGaAlAs ridge waveguide electro-absorption modulated laser with integrated SOA. Phys Status Solidi. 2016;A213:970-4

7. Theurer M, Zhang H, Wang Y, Chen W, Zeng L, Troppenz U, Przyrembel G, Sigmund A, Moehrle M, Schell M. $2 \times 56$ GB/s from a double side electroabsorption modulated DFB laser and application in novel optical PAM4 generation. J Lightw Techn. 2017;35(4):706-10.

8. Theurer M, Moehrle M, Troppenz U, Bach H-G, Sigmund A, Przyrembel G, Gruner M, Schell M. 4 × 56 Gb/s high output power electroabsorption modulated laser array with up to 7km fibre transmission in L-band. J Lightw Techn. 2018;36(2):181-6.

9. Baier M, et al. $112 \mathrm{~Gb} / \mathrm{s}$ PDM-PAM4 generation and $80 \mathrm{~km}$ transmission using a novel monolithically integrated dualpolarization electro-absorption modulator InP PIC. Proc ECOC. 2017;2017:1-3.

10. Baier M, Soares FM, Gaertner T, Schoenau A, Moehrle M, Schell M. New Polarization Multiplexed Externally Modulated Laser PIC, ECOC 2018 (Rome, Italy), paper Mo4C.2; 2018.

11. Baier M, Soares FM, Gaertner T, Moehrle M, Schell M. Fabrication tolerant integrated polarization rotator design using the Jones Calculus. J Lightw Technol. 2019;37(13):3106-12.

12. Morsy-Osman MH, Chagnon M, Plant DV. Four-dimensional modulation and stokes direct detection of polarization division multiplexed intensities, inter polarization phase and inter polarization differential phase. J Lightw Technol. 2016; 34:1585-92.

13. M. Baier, F.M. Soares, A. Schoenau, Y. D Gupta, D. Melzer, M. Moehrle, M. Schell: Fully integrated Stokes Vector Receiver for 400 Gbit/s", OFC 2019 (San Diego), paper Tu3E.2 (2019).

14. F. M. Soares et al.: High-Performance InP PIC Technology Development based on a Generic Photonic Integration Foundry, OFC 2018, paper M3F.3 (2018).

15. K. Shi et al.: System Demonstration of Nanosecond Wavelength Switching with Burst-mode PAM4 Transceiver", ECOC 2019, paper PDP 3.7 (2019).

\section{Publisher's Note}

Springer Nature remains neutral with regard to jurisdictional claims in published maps and institutional affiliations.

\section{Submit your manuscript to a SpringerOpen ${ }^{\circ}$ journal and benefit from:}

- Convenient online submission

- Rigorous peer review

- Open access: articles freely available online

- High visibility within the field

- Retaining the copyright to your article

Submit your next manuscript at $\boldsymbol{\nabla}$ springeropen.com 